\title{
Brain Tumor Extraction and Classification from MRI Images
}

\author{
Smriti Bhatnagar, Shubham Sharma
}

\begin{abstract}
This paper proposes a methodology in which detection, extraction and classification of brain tumour is done with the help of a patient's MRI image. Processing of medical images is currently a huge emerging issue and it has attracted lots of research all over the globe. Several techniques have been developed so far to process the images efficiently and extract out their important features. The paper describes certain strategies including some noise removal filters, grayscaling, segmentation along with morphological operations which are needed to extract out the features from the input image and SVM classifier for classification purpose.
\end{abstract}

Keywords: Grayscaling, MRI, Morphological operations, MATLAB, Segmentation, SVM Classifier

\section{INTRODUCTION}

A brain tumour is a lump of tissues which is caused when brain cells divide and grow in an uncontrolled way. There are certain ways like exposure to harmful radiation or intake of harmful substances which can trigger cells to behave abnormally causing the formation of a cluster of cells known as a tumour. Tumours can be really harmful sometimes if they grow abnormally and suppress any other brain nerve. Tumours have been classified as Benign tumour i.e. non-cancerous tumour and Malignant tumour i.e. a cancerous tumour. [1]

A benign tumour can be understood as a non-cancerous tumour which does not invade into other surrounding tissues and does not spread into other parts of the body also. Hence benign tumours are generally do not considered as harmful or life-threatening but they can be problematic if they press on vital parts of brain and blood vessels. Therefore, sometimes they require treatment but otherwise, doctors may simply prefer waiting to make sure they cause no problem. Surgery is a common treatment for brain tumours. A benign tumour usually doesn't recur after removal and if they do then it is usually at the same place Factors which can be linked for the origin of benign tumours include environmental toxins, genetics, radiation, diet, stress, injury or any infection.

A malignant tumour is a cancerous type of tumour which can invade to other body organs also. Metastasis is a state in which blood stream gets filled with cancerous cells and thus there is threat of cancer cells reaching other vital tissues and body organs as well. These type of tumours grow rapidly at a fast pace and hence requires advance treatment and proper medication as well. If not treated at early stages, malignant

Revised Manuscript Received on July 10, 2019.

Smriti Bhatnagar, Electronics and Communication Department, Jaypee Institute of Information Technology, Noida, U.P, India.

Shubham Sharma, Electronics and Communication Department, Jaypee Institute of Information Technology, Noida, U.P, India. tumours can also become a threat to life. They can recur in different parts also after removal. The cells involved in a malignant tumour are usually of abnormal shape. Their symptoms include weight loss, severe pain in the affected region and fatigue.

Magnetic Resonance Imaging abbreviated as MRI is a term which can be understood as an advances technique for medical imaging purposes. Implementation of this technique can be found worldwide and very high-quality scan results are being produced successfully using MRI methodology.. This technique involves the use of a strong magnetic field and radio waves to produce high-resolution output images. These high-quality images help in an easy derivation of detailed anatomical information to examine and discover abnormalities in the tumour affected part. [1]

The initial steps which are generally needed to make the input image more readable include steps like de-noising of image and its enhancement for better contrast. The pre-processing is followed by segmentation and morphological operations for tumour detection and extraction purposes. If the presence of a tumour is confirmed then the classification algorithm is applied with the help of SVM classifier.

\section{METHODOLOGY}

The proposed work can be split into two parts. The first part includes processing of input image which include several steps described below to detect and extract out tumour region. The second part involves the classification of the tumour into a benign and malignant tumour. SVM Classifier is used to determine the category of the tumour based on training dataset results. Steps of the algorithm are as follows:

\section{A. Anisotropic Diffusion Filter}

This filtering technique aims at reduction of the noising features available in the image. The main objective here is to suppress these noisy pixels without disturbing the important features required for the detection purposes. Here the image generates a parameterized family of successively more and more blurred images based on the diffusion process. Every image which is given as input to Anisotropic diffusion filter is processed by convolving Gaussian filter with ht original input image's pixel matrix. The behaviour of Gaussian filter is 2-dimensional where increasing parameters results in increase of the filter's width simultaneously. Hence it can be stated that MRI image's space-variant transformation is done using anisotropic diffusion filter which is actually non-linear in behaviour. 
This filtering technique offers the advantage of removing noise and enhancing quality without blurring edges. This diffusion technique is also known as Perona-Mallik diffusion because Pietro Perona and Jitendra Malik developed this idea and their work revolved around two main function which they proposed in 1990. These functions are important for determining the diffusion constant.

$c(\|\Delta\|)=e^{-\left(\frac{\| \Delta \Delta}{K}\right)^{2}}$

$\&$

$$
c(\|\Delta\|)=\frac{1}{1+\left(\frac{1 \Delta}{\Sigma}\right)^{2}}
$$

Here, the $\mathrm{K}$ constant represents the sensitivity factor and is generally identified with the help of experimental results.

The proposed diffusion filter works on three parameters smoothing constant, number of iteration and size of adjacent relation. Size o adjacent relation defines a trade-off between precision and computational effort. The other two parameters are helpful in determining the result in terms of the image under and overshooting.

\section{B. Grayscaling}

Grayscaling is a method to convert continuous tone image to an image which a computer can easily manipulate. The continuous-toned image uses a huge number of shades of gray which becomes really complex for computation. A grayscale image has the value of each pixel as a single sample which represents a particular amount of light based on the weighted combination of frequencies or wavelength. The brightness of the pixel is can be represented by the value associated with it. Complete absence of the light which was transmitted is represented with the black colour which is the darkest shade in a grayscale image. In contrast, the full transmitted or reflected light is displayed with the help of white shade in a pixel. [3]

\section{Threshold Segmentation}

Threshold segmentation as the name suggests is based on threshold value for segmenting image. This approach is very simple to implement. This technique is very useful for conversion into binary image from a grayscale image based on some predefined clip-off value. [1] The main objective of the specified approach is simplifying and changing the representation of an image into something more meaningful which is easier to analyze. Image segmentation helps in locating objects and boundaries in the image in order to find the tumour region. The basic idea behind threshold segmenting is to group together pixels depicting same properties under same label name for easier analysis. [1]

The segmented obtained thus contains small portions of segments of pixels with same characteristics such that they together can covers the entire image. The features upon which pixels are labelled can be based on area, texture or intensity.

\section{Morphological Operations}

The group of some non-linear operations is collectively termed as morphological operations. These operations are based on extracting shapes and features from MRI images.
[2] Pixels are ordered for these operations and hence it can be easily applied on binary images. The key element for this method is a structuring element which is like a small shape or template used for probing the image.

A structuring element consists of pixel matrix with values zeros and ones only. The structuring element is used for comparison purposes with its adjacent pixels throughout the image. With the help of these operations, a new image is developed in which non-zero pixel values corresponds to the successful match of structuring element at that position. Thus morphological operations are based on 'fits' or 'hits' criteria i.e. structuring elements fits within neighborhood or intersects the neighborhood correspondingly. [2]

Based on these operations, the tumour region is identified. If tumour region is detected is present then further processing is done in order to classify the tumour into benign or malignant but if not then there is no presence of the tumour.

\section{E. Discrete Wavelet Transform}

Feature extraction is a necessary part of the classification of a tumour and for this purpose, a discrete wavelet transform is used. A DWT model is one amongst the foremost vital image analysis models that is intended for cascaded filtering model with sub-sampling factors in varied forms. [3] Multilevel wavelet decomposition is applied in this proposed work in 3-level and output is obtained in sub-bands.

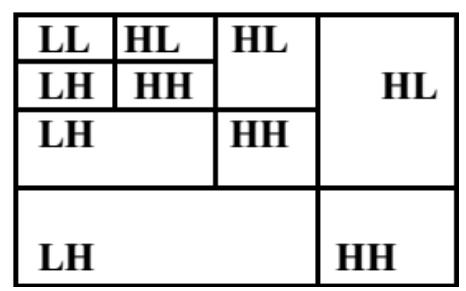

Fig. 1 Pyramidal 3-level wavelet decomposition

Daubechies wavelet is the most generally utilized discrete wavelet change strategy introduced by Belgian mathematician Ingrid Daubechies in 1988. Her findings figured the utilization of repeat relations to produce dynamically better discrete samplings of a certain mother wavelet work where every goals is twice that of the past scale. A series of filters is required to generate wavelet transform of a signal $x(n)$. First of all, the image is processed with impulse response $g$ resulting in a convolution of the two as shown below.

$$
y[n]=(x * g)[n]=\sum_{k=-\infty}^{m} x[k] g[n-k]
$$

Simultaneous decomposition is also done with the help of a high pass filter denoted as $h$. The yield of dwt gives detailed coefficient which is resultant from high pass channel and estimation coefficient which is resultant from low pass channel. These filters are also known as quadrature mirror filter 


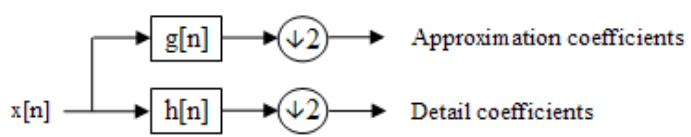

Fig. 2 Filter Analysis

Since half the frequencies are removed after first processing the signal is again passed to high and low pass filters but with the cut-off frequency one halved this time.

\section{F. Extraction of features}

The factual technique for finding the surfaces to consider the spatial association of the pixels is Gray Level Co-Occurrence Matrix abbreviated as GLCM. [4] The target of GLCM is to recognize the outside of a picture by assessing the pixel in pair with explicit qualities and determined spatial relationship. GLCM helps in estimating the factual extricated parameters. This strategy is broadly acknowledged on account of its raised rightness and a littler measure of work out time. GLCM contains the subtleties with reference to the situation of pixels having related values of grey level. The extracted features are as follows. [5]

\section{Contrast}

Contrast represents the difference between the maximum and minimum pixel intensity in an image. A picture with high differentiation will display a full scope of tones from dark to white, with enhanced features and dull shadows.

\section{Correlation}

Correlation displays the extent to which two or more pixel values fluctuate together. It measures image linearity.

\section{Energy}

It is a texture to detect the homogeneity changing and to mirror the dissemination of input image's consistency of texture and weights.

\section{Homogeneity}

Homogeneity in image interprets the regions on the image with no rough transitions like edges or corners, instead of smooth regions with presumably same or similar colour values.

\section{Mean}

It is one of the basic statistical parameters. It is like a framework which utilizes concepts of masking over every pixel in the image. Every segment of the pixels which comes under the mask is grouped together to represent a single pixel value and this grouping is based on average values of all pixels inside mask.

Mean $=\frac{1}{N} \sum_{i=1}^{N} X i$

Where $\mathrm{N}$ is the number of pixels inside the window and $\mathrm{Xi}$ represents each pixel.

\section{Standard Deviation}

Standard deviation is used to calculate the deviation among pixels. An average pixel value is used to find the variation of other remaining pixels with the chosen average pixel. Mathematically standard deviation is given by:

$$
f(x, y)=\sqrt{\frac{1}{m n-1} \sum_{(r, c) \in W}\left(g(r, c)-\frac{1}{m n-1} \sum_{(r, c) \in W} g(r, c)\right)^{2}}
$$

Here ' $g$ ' represents the input image, Restored image is presented by $f(x, y), m x n$ is the size of the window and ' $r$ ' and ' $\mathrm{c}$ ' signifies the rows and column values respectively.

\section{Entropy}

The extent of randomization of intensity distribution inside image is termed as Entropy.

Entropy $=-\sum_{i} P i \log _{2} P i$

Here $P_{i}$ is the probability of difference among two adjacent pixels being equivalent to $i$.

\section{RMS}

The root-mean-square deviation is used to measure the difference between the actual and predicted pixel values. The RMSD of an estimator $\hat{\theta}$ with respect to an estimated parameter $\theta$ is given as

$$
\operatorname{RMSD}(\hat{\theta})=\sqrt{E\left((\hat{\theta}-\theta)^{2}\right)}
$$

\section{Skewness}

The dissymmetry in the pixel's probability distribution is measured by skewness. It can be positive or negative. Mathematically, skewness can be given by:

$f(x, y)=\sqrt{\frac{\sum_{i=1}^{1}\left(x_{i}-x\right)^{3}}{(n-1) s^{3}}}$

Where, $x_{i}$ is the current observation, $\mathrm{x}$ is the mean, $\mathrm{n}$ shows the total number of observations and $\mathrm{s}$ represents variance.

\section{Variance}

The variance is a measure of how far a set of numbers is spread out. It is one of several descriptors of a probability distribution, describing how far the numbers lie from the mean value.

The variation shows the proportion of spreading of pixel values. It depicts how far the values lie from the mean esteem. Mathematically variance is given by:

$f(x, y)=\frac{1}{m n-1} \sum_{(r, c) e W}\left(g(r, c)-\frac{1}{m n-1} \sum_{\left(r_{u} c\right) e W} g(r, c)\right)^{2}$

Here ' $g$ ' represents the input image, Restored image is presented by $f(x, y), m x n$ is the size of the window and ' $r$ ' and ' $\mathrm{c}$ ' signifies the rows and column values respectively.

\section{Smoothness}

It is a process of producing image with pixels of lower values. In this process, noises are removed from the image and it is passed through low pass filter. 


\section{Kurtosis}

Here for a real valued random variable, the probability distribution curve is measured. Longer and fatter tails are resultant of a high kurtosis distribution and short and thin tails with rounded peak are features of a low kurtosis.

Mathematically kurtosis is given as follows:

$f(x, y)=\sqrt{\frac{\sum_{i=1}^{n}\left(x_{i}-x\right)^{4} / N}{s^{4}}}$

Where, $x_{i}$ is the current observation, $\mathrm{x}$ is the mean, $\mathrm{n}$ represents number of observations and $\mathrm{s}$ depicts variance.

\section{Inverse difference movement}

Inverse Difference Moment abbreviated as IDM is used to determine texture of an image. Determination of local homogeneity of input image is done under IDM. It quantifies the closeness of the dispersion of the GLCM components to the diagonal of GLCM.

\section{SVM CLASSIFIER}

Support Vector Machine Classifier is used to classify classes based on a hyper-plane which is similar to a line cutting a plane into two parts and hence also known as a discriminative classifier. [4] Each class is on either side of the hyper plane. Thus SVM is used when the input data is to be classified among these classes only. The classification is done by finding the appropriate hyper-plane distribution line which segregates values of one class from another class. The hyper-plane with largest margin between the two classes can be considered best. The below figure depicts the structure of SVM classifier.

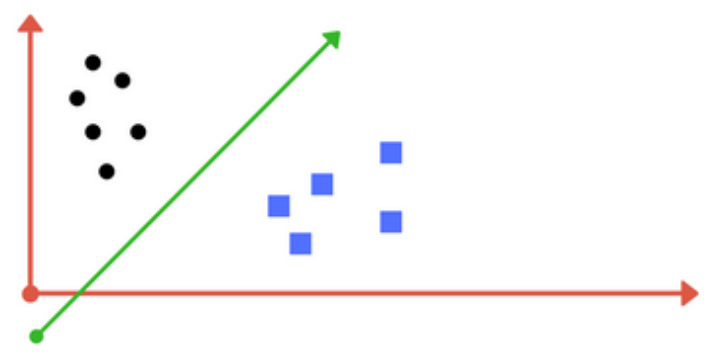

Fig. 3 SVM Classifier

\section{EXPERIMENTAL RESULTS}

This section presents the simulation results depicting the performance of the proposed algorithm. The following figures are the results of various filters and operations applied to the input image as shown.

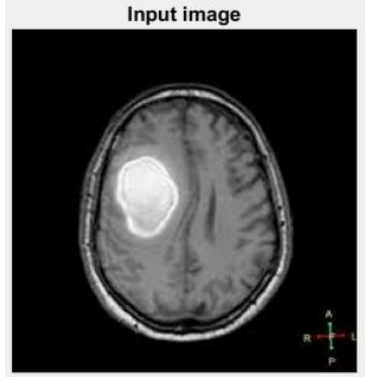

Fig. 4 MRI Image of tumour affected brain

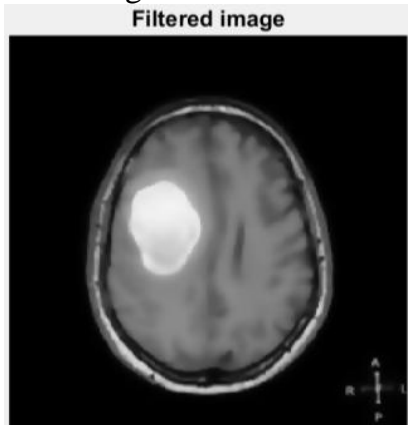

Fig. 5 Anisotropic Diffusion filtered image

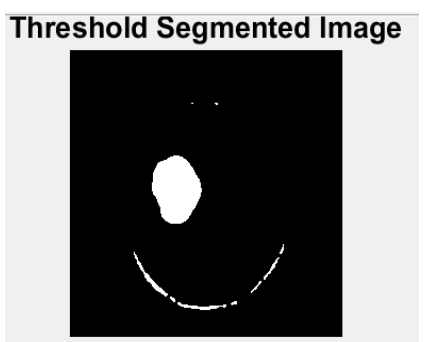

Fig. 6 Threshold Segmented Image

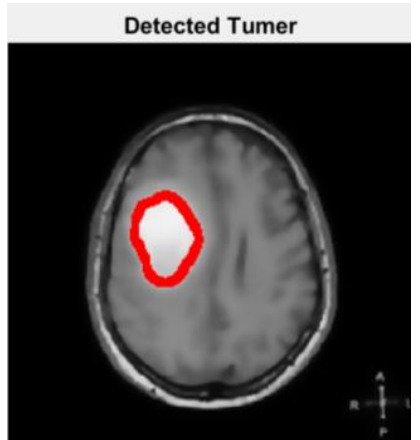

Fig. 7 Detected Tumour Image

For a tumour less image a message was displayed as "no tumour". Similarly, messages were displayed for images with a benign tumour and malignant tumour as shown. 


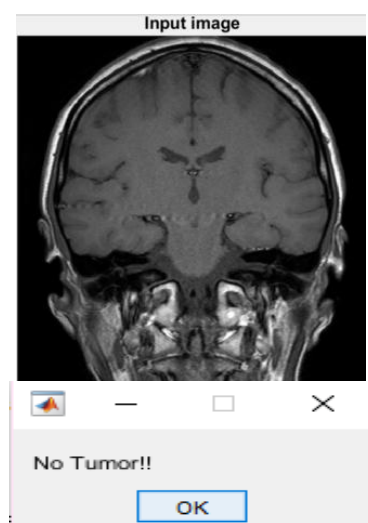

Fig. 8 (a) Input Image (b) Message box displaying "No Tumour"

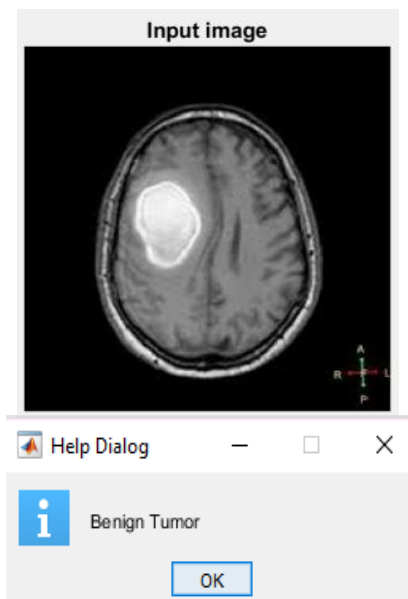

Fig. 9 (a) Tumour Detection (b) Message displaying "Benign Tumour"

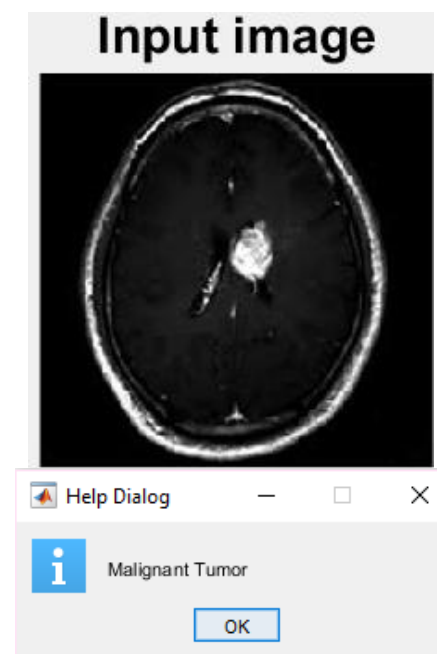

Fig 10 (a) Tumour Detection, (b) Message displaying "Malignant Tumour"

\section{CONCLUSION}

The proposed algorithm is applied on a dataset of 40 images out of which 6 images were diagnosed without any tumour. Out of the remaining 34 images, the algorithm diagnosed 18 images with the presence of Benign tumour and remaining 16 images with Malignant tumour with the help of SVM Classifier based on a predefined training set.
But the actual diagnosed sheet showed that the non-tumour images were 4 and the images with presence of Benign tumour and Malignant tumour were 19 and 17 respectively. Hence the comparison chart between predicted and actual results is as follows.

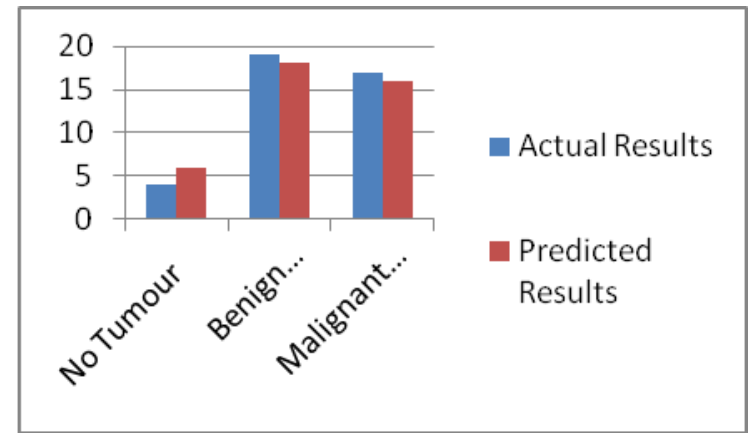

Fig 11 Comparison Chart

Based on the results obtained, it can be concluded that the proposed algorithm displayed an accuracy of $95 \%$ for the particular dataset.

\section{REFERENCES}

1. Ehab F. Badran, Esraa Galal Mahmoud, and Nadder Hamdy, "An Algorithm for Detecting Brain Tumours in MRI Images" Department of Electronics and Communications Engineering Arab Academy for Science and Technology \& Maritime Transport Alexandria, November 2010.

2. Chang Wen Chen, Jiebo Luo and Parker, K.J, "Image segmentation via adaptive K-mean clustering and knowledge-based morphological operations with biomedical applications", IEEE Transactions on Image Processing, Vol. 7 , Issue 12, 1998.

3. W. Gonzalez, "Digital Image Processing", 2nd ed. Prentice Hall, Year of Publication 2008, Page no 378.

4. Y. Zhang, L. Wu, "An MRI Brain Image Classifier via Principal Component Analysis and Kernel Support Vector Machine", Progress In Electromagnetic Research, Vol. 130, 369-388, 2012.

5. Vijay Kumar and Priyanka Gupta, "Importance of Statistical Measures in Digital Image Processing", International Journal of Emerging Technology and Advanced Engineering, Volume 2, Issue 8, August 2012.

\section{AUTHORS PROFILE}

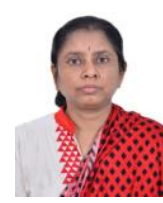

Smriti Bhatnagar has obtained her Master's degree . from MNNIT, Allahabad and is currently working as Assistant Professor in ECE Deptt. JIIT, NOIDA. She is a fellow member of IETE and a member of IEEE.

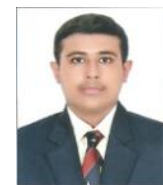

Shubham Sharma is currently pursuing his Bachelor of Technology degree from Jaypee Institute of Information Technology, Noida 